Original article

\title{
Evaluation of field recognizable morbidity indicators of Schistosoma haematobium infection among primary school children in Ghana
}

\author{
Ato Kwamena Tetteh ${ }^{1}$, Joseph Otchere ${ }^{2}$, Langbong Bimi ${ }^{3}$, Irene Ayi ${ }^{2}$ \\ ${ }^{1}$ Anglican University College of Technology, Nkoranza, Brong Ahafo Region, Ghana \\ ${ }^{2}$ Noguchi Memorial Institute for Medical Research University of Ghana, Legon, Accra, Ghana \\ ${ }^{3}$ University of Ghana, Legon, Accra, Ghana
}

Received 17 February 2016, Revised 16 March 2016, Accepted 12 April 2016

(C) 2016, Tetteh A.K., Otchere J., Bimi L., Ayi I.

(C) 2016, Russian Open Medical Journal

Abstract: Aim - This study was a retrospective evaluation of morbidity indicators and the use of questionnaire in decisions that relate to Schistosoma haematobium infection control among primary school children in Ghana.

Materials and Methods - In all, 417 pupils in three primary schools (Kasseh sub-District, Dangme East District, Ghana), provided urine samples, which were examined for $S$. haematobium ova. The study utilized a structured questionnaire, the Urit $10 \mathrm{~V}$ multi-stick urine reagent strips and the filtration method.

Results - Overall, prevalence by microscopy was $20.9 \%$ (requires targeted treatment once every two years), and was higher (14.0\%) among the $10-15$ years age group $\left(\chi^{2}=22.44, p<0.001\right)$. The lowest prevalence was among the $<6(0.5 \%, 2 / 417)$ and $\geq 19(1.4 \%, 6 / 417)$ years age groups. There was no significant difference in prevalence among females $(7.9 \%, 33 / 417)$ and males $(12.9 \%, 54 / 417)\left(\chi^{2}=2.58, p=0.108\right)$. Self-reported haematuria was $43.2 \%$ (targeted treatment once a year). There was a significant difference between female (16.1\%, 67/417) and male $(27.1 \%, 113 / 417)$ responses to self-reported haematuria $\left(\chi^{2}=4.170, p<0.001\right)$. A lower consolidated mean prevalence of $11.7 \%$ was estimated among pupils who had ova in their urine and also exhibited three morbidity indicators (haematuria, leucocyturia and proteinuria). Combined and averaged sensitivity and specificity estimations (sensitivity $=55.4 \%$, specificity $=67.8 \%$, Positive Predictive Value $(P P V)=53.4 \%$, Negative Predictive Value $(N P V)=64.2 \%$ ) of these morbidity indicators, was insufficient in equalizing the reliability of ova quantification. With the exception of leucocyturia, which was common among all pupils, $28.75 \%$ (25/87) of the infected individuals did not have any form of haematuria or proteinuria. Out of these, one pupil had heavy ova intensity ( 80 eggs/10ml of urine) while the remaining 24 had low ova intensity infection (1-44 eggs/10ml of urine). Questionnaire correctly identified $56.3 \%$ of pupils with S. haematobium ova in their urine, also reporting bloody urine (PPV=27.2\%; NPV=84.0\%). It also identified $49.4 \%$ of pupils with ova, reporting painful urination ( $P P V=26.1 \%, N P V=82.5 \%)$.

Conclusion - This study emphasizes the relevance of evaluating the criteria for interpretation of morbidity indicators, prior to their field application. Questionnaire use remains key for surveillance purposes, to determine the presence of the infection in an area. Future studies should consolidate similar data elsewhere in Ghana for a better understanding of morbidity indicators and questionnaire use for decisions that relates to control strategies.

Keywords: Schistosoma haematobium, school children, Ghana, questionnaire, diagnosis, haematuria

Cite as Tetteh AK, Otchere J, Bimi L, Ayi I. Evaluation of field recognizable morbidity indicators of Schistosoma haematobium infection among primary school children in Ghana. Russian Open Medical Journal 2016; 5: e0303.

Correspondence to Mr. Ato Kwamena Tetteh. Address: Department of Community Medicine and Health, Anglican University College of Technology, P.O. Box 78, Nkoranza, B/A, Ghana. E-mail: kwamena.atetteh@angutech.edu.gh

\section{Introduction}

Available data indicate that urinary schistosomiasis is endemic in Ghana [1, 2]. Consistently, intermediate host snail elimination has not been cost effective, due to the negative impact molluscicides have on the ecosystem [3]. Contemporary initiatives to reduce the burden in Ghana and globally is largely on praziquantel use, depending on the prevalence obtained in a particular area [4]. Prevalence data, currently, is based on detection of parasite ova in urine. Other studies have shown varying trends with morbidity indicators (haematuria, proteinuria and leucocyturia) as being comparable to microscopy in rural settings. In Ghana and elsewhere, macro and microhaematuria are known to be the major indicators of infection with Schistosoma haematobium [5-8]. The use of questionnaires has been described as rapid, relatively inexpensive, and is not markedly inferior to other techniques [5, 9-11]. This is explained by the fact that S. haematobium infection is easily perceived through haematuria. It is ranked as one of the best options for field use in surveillance, mass treatment and epidemiological studies. A major challenge of bias arise due to the stigma attached to passing blood in urine in the peri-urban and urban areas, making it difficult for people to respond truthfully, even when the symptoms are present. The 
usefulness of questionnaire (especially, asking study participants whether they see blood in their urine) as a diagnostic tool in the estimation of prevalence has not been exploited in Ghana. This is crucial, also with regards to other developing countries, given the limited resources, logistical and technical constraints associated with estimating prevalence by microscopy.

This current study explored the reliability of three morbidity indicators and questionnaire in determining prevalence of urinary schistosomiasis in the Kasseh East sub-District (Ghana).

\section{Material and Methods \\ Study area}

The study was conducted in three primary schools located in three different communities, Afiadenyigba, Dorgobom and Tojeh in the Kasseh sub-District, of the Dangme East District (Figure 1). The district is less than $100 \mathrm{~km}$ East of Accra, and is typically coastal savannah, consisting of grass with isolated patches of scrubs and trees. The area is characterized with very low humidity and has the least precipitation in the coastal zones of Ghana. Yearly precipitation peaks (April to June and September to October) for the area range between 0.740 and $0.900 \mathrm{~m}$. The highest mean monthly temperature is $303.15^{\circ} \mathrm{K}$ (March-April), and the lowest $299.15^{\circ} \mathrm{K}$ (August).

\section{Study design and population}

The study was cross-sectional, and pupils were recruited by voluntary participation after informed consent had been obtained from their teachers and parents with their assent. Urine samples were collected and examined for S. haematobium ova. In all, 206 pupils from Afiadenyigba, 95 from Dorgobom and 116 from the Tojeh District Assembly (D/A) Primary Schools consented and participated in the study.

\section{Ethical clearance}

Ethical clearance was obtained from the Institutional Review Board (IRB) of the Noguchi Memorial Institute for Medical Research (NMIMR).

\section{Questionnaire administration}

Personal information including name, sex, age, and place of residence were collected by questionnaire interview (Appendix 1). Questionnaires also obtained information on their knowledge, practices, risk of exposure to infection and treatment history for urinary schistosomiasis.

\section{Collection and examination of samples}

Urine samples were collected in appropriately labeled sterile containers with screw lids. Containers were given to pupils for collection the same day between the hours of 11:00 and 14:00 hrs GMT when egg excretion is known to be highest [12]. These were kept in an ice chest and transported to the laboratory for processing and examination within 24 hours. Samples were visually assessed for macrohaematuria, followed by the use of Urit 10V multi-stick urine reagent strips (Urit Medical Electronic Company Ltd) to detect microhaematuria, leucocyturia and proteinuria [13]. Egg/10 $\mathrm{ml}$ of urine for $S$. haematobium was determined and categorized into low and high intensity [4].

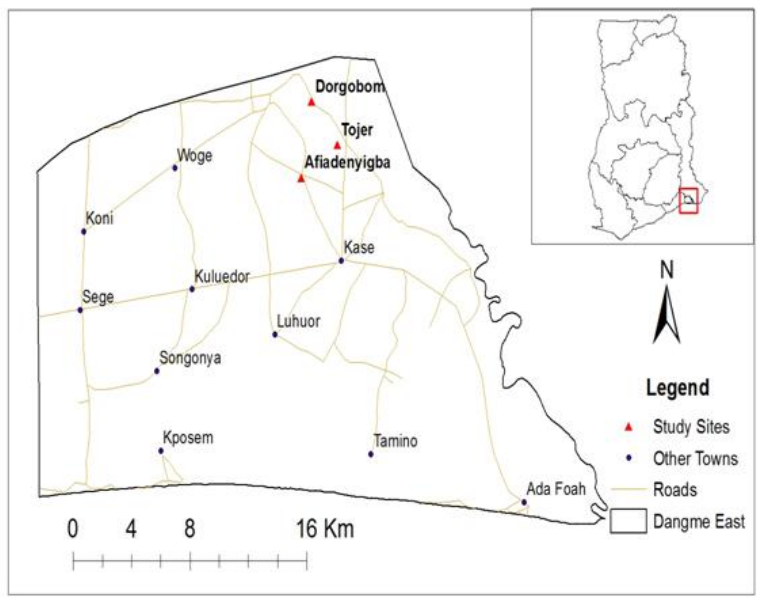

Figure 1. Map of Dangme East District, Ghana

\section{Data analysis}

Questionnaire, morbidity indicators and parasitological data were computerized using SPSS (Version20.0: SPSS Inc, Chicago IL). Questionnaire responses were analysed for level of knowledge, practices, risk of exposure to infection and treatment history, whilst the prevalence of parasites detected were determined by frequencies. Associations between parasitic infections and their risk factors were estimated using Prevalence Odds Ratios (PORs). Chi-Square $\left(\chi^{2}\right)$ and $p$-values (5\% error margin) were estimated to show the magnitude of differences in associations. The diagnostic performance of questionnaire and the semi-quantitative methods were assessed by doing a cross classification with the gold standard, and manually calculated Sensitivity, Specificity, Positive and Negative Predictive Values (PPV and NPV) using the following formulas:

- $\quad$ Sensitivity $=T P /(T P+F N)$

- $\quad$ Specificity $=T N /(T N+F P)$

- $\quad$ Positive Predictive Value (PPV) $=\mathrm{TP} /(\mathrm{TP}+\mathrm{FP})$

- $\quad$ Negative Predictive Value (NPV) $=T N /(T N+F N)$

where, $\mathrm{T}$ is true, $\mathrm{F}-$ false, $\mathrm{P}-$ positive, and $\mathrm{N}$ - negative.

\section{Results}

\section{Demographics of participants}

Overall, 417 pupils (class 1-6) from three District Assembly (D/A) Primary Schools participated in the study from September, 2007 to August, 2008. This comprised 54.4\% (227/417) males and $45.6 \%$ (190/417) females. Ages ranged from 3 to 26 years, with an overall mean of $11.3 \pm 3.4$ years (mean \pm standard deviation) (Table 1).

\section{Prevalence by microscopy}

Out of the total, $20.9 \%$ (87/417) had parasite ova (Figure 2). There was no significant difference in prevalence among the three schools $\left(\chi^{2}=7.638, p=0.022\right)$. Of the number positive, $7.9 \%$ $(33 / 417)$ were females while $12.9 \%$ (54/417) were males $\left(\chi^{2}=2.582, p=0.108\right)$ (Table not shown). 
Table 1. Details of participants

\begin{tabular}{|c|c|c|c|c|c|}
\hline D/A primary school & No. involved (\%) & Age range, years & Males, no. (\%) & Females, no. (\%) & Mean age, years \\
\hline Afiadenyigba & $206(49.4)$ & $3-26$ & $111(26.6)$ & $95(22.8)$ & 12 \\
\hline Dorgobom & $95(22.8)$ & $5-20$ & $45(10.8)$ & $50(12.0)$ & 11 \\
\hline Tojer & $116(27.8)$ & $5-20$ & $71(17.0)$ & $45(10.8)$ & 11 \\
\hline Overall & $417(100)$ & $3-26$ & $227(54.4)$ & $190(45.6)$ & 11.3 \\
\hline
\end{tabular}

D/A, District Assembly.

Table 2. Sensitivity and specificity estimations for semi-quantitative methods

\begin{tabular}{|c|c|c|c|c|c|}
\hline Diagnositic indicator & Prevalence by semi-quantitative tests*, \% (no./total) & Sensitivity, \% & Specificity, \% & $P P V, \%$ & $N P V, \%$ \\
\hline Macrohaematuria & $2.9(12 / 417)$ & 13.6 & 99.1 & 80.0 & 81.1 \\
\hline Microhaematuria & $10.8(45 / 417)$ & 40.9 & 95.2 & 69.2 & 85.8 \\
\hline Leucocyturia & $20.9(87 / 417)$ & 100 & 0 & 21.1 & 0 \\
\hline Proteinuria & $13.9(58 / 417)$ & 67.0 & 76.7 & 43.4 & 89.7 \\
\hline
\end{tabular}

* Urinalysis parameters are for participants who were also ova positive.

PPV, Positive Predictive Value; NPV, Negative Predictive Value.

Table 3. S. haematobium ova intensity with presence of morbidity indicators

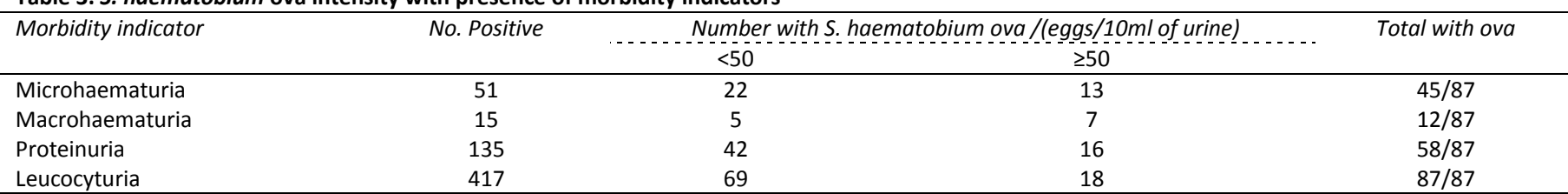

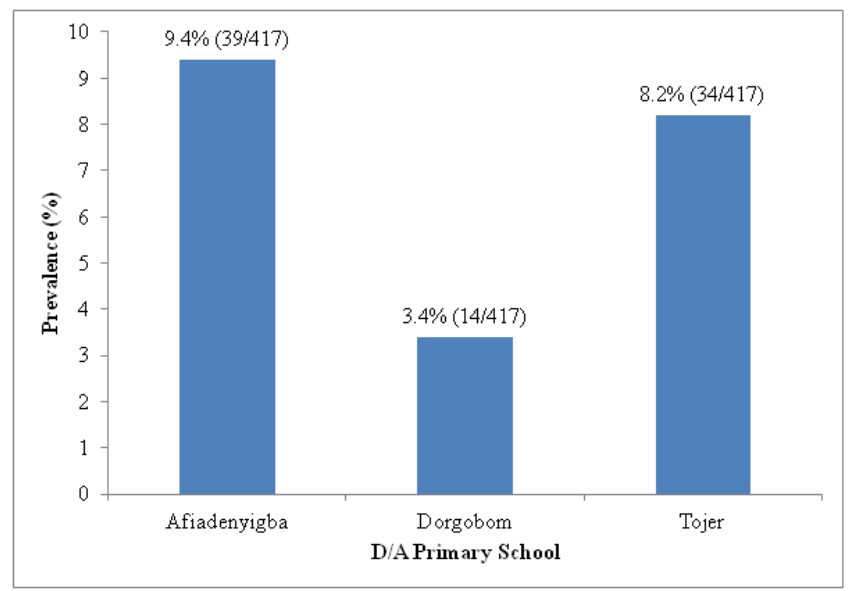

Figure 2. Prevalence of $S$. haematobium infection among the three D/A Primary Schools

With regards to prevalence among age groups, the 10-12 and the 13-15 years age groups had a prevalence of $7.0 \%$ (29/417) each. The remaining age groups had prevalence below $3 \%$ (Table 4). The lowest prevalence occurred among the $<6(0.5 \%$, $2 / 417)$ and $\geq 19(1.4 \%, 6 / 417)$ years age groups.

\section{Sensitivity and specificity of semi-quantitative methods}

Participant's microscopy results were matched with the urine chemistry tests for comparison. Of the total, $2.9 \%(12 / 417)$ had macroscopic blood and as well had ova in their urine. Urine chemistry tests performed showed that $10.8 \%$ (45/417) had microhaematuria, and ova as well (Sensitivity $=40.9 \%$; Specificity $=$ 95.2\%; PPV $=69.2 \% ; \mathrm{NPV}=85.8 \%$ ). All participants parasite ova were positive for urinary leucocytes (Sensitivity $=100.0 \%$;
Specificity $=0.0 \% ; \mathrm{PPV}=21.1 \% ; \mathrm{NPV}=0.0 \%)$. Proteinuria was also detected in $13.9 \%$ (58/417) of the participants who also had ova (Table 2). Further deductions indicates that, of the ova positive individuals, $86.4 \%(76 / 87)$ had no macrohaematuria, $33.3 \%$ $(29 / 87)$ had no proteinuria, while $59.8 \%$ (52/87) were also negative for microhaematuria.

Intensity of S. haematobium ova and the presence of morbidity indicators

Of the total that was ova positive, $79.3 \%(69 / 87)$ were in the low intensity category, while the remaining $20.7 \%$ (18/87) were of high intensity. A total of 6,469 eggs $/ 10 \mathrm{ml}$ of urine were counted among the 87 participants (an average of 74 eggs $/ 10 \mathrm{ml}$ of urine per pupil). Highest ova count per individual was $2,179 \mathrm{eggs} / 10 \mathrm{ml}$ of urine, while the least was $1 \mathrm{egg} / 10 \mathrm{ml}$ of urine. The number of low and high intensity infections peaked among 10-12 and 13-15 years age groups. The In all, 45 pupils were microhaematuria positive and also positive by microscopy. All ova positive participants also had leucocyturia (Table 3). Of the number positive, 25 had no haematuria and proteinuria. Of these, 24 were of low intensity, while one (1) was heavily infected.

\section{Analysis of selected questions}

The four major questions considered for analysis are as follows:

\section{W - "Do you know that urinating blood is a disease"?}

Overall, $42.2 \%(176 / 417)$ knew that passing blood in ones urine is a disease. Of this number, $29.0 \%(51 / 176)$ were positive by microscopy (Table 4). Out of the remaining 241 who responded "no" to this question, $14.9 \%$ (36/241) were positive by microscopy (data not shown). 
Table 4. Selected questionnaire responses according to age group

\begin{tabular}{|c|c|c|c|c|c|c|c|c|c|c|c|}
\hline \multirow[t]{2}{*}{ Age, years } & \multirow[t]{2}{*}{ No. involved } & \multicolumn{2}{|c|}{$W$} & \multicolumn{2}{|c|}{$x$} & \multicolumn{2}{|c|}{$Y$} & \multicolumn{2}{|c|}{$Z$} & \multicolumn{2}{|c|}{ Microscopy $(N=417)$} \\
\hline & & Yes & No. +ve & Yes & No. +ve & Yes & No. +ve & Yes & No. +ve & ${ }^{\&}$ No. +ve & $\%$ \\
\hline$<6$ & 26 & 4 & 1 & 6 & 1 & 12 & 1 & 11 & 1 & 2 & 0.5 \\
\hline $7-9$ & 99 & 15 & 3 & 31 & 3 & 31 & 3 & 29 & 3 & 9 & 2.2 \\
\hline $10-12$ & 138 & 56 & 15 & 44 & 8 & 58 & 12 & 49 & 8 & 29 & 7.0 \\
\hline $13-15$ & 106 & 66 & 19 & 33 & 8 & 52 & 21 & 49 & 19 & 29 & 7.0 \\
\hline $16-18$ & 37 & 29 & 10 & 7 & 2 & 21 & 9 & 21 & 9 & 12 & 2.9 \\
\hline$\geq 19$ & 11 & 6 & 3 & 3 & 0 & 6 & 3 & 6 & 3 & 6 & 1.4 \\
\hline Total & 417 & 176 & 51 & 124 & 22 & 180 & 49 & 165 & 43 & 87 & 20.9 \\
\hline
\end{tabular}

WXYZ represents selected questions analyzed for this manuscript.

Yes - Number of participants who responded "yes" to questions WXYZ.

No. +ve - Number positive by microscopy who responded "yes" to questions WXYZ.

${ }^{\&}$ No. +ve - Number positive by microscopy alone among the age groups.

W - "Do you know that urinating blood is a disease"?

$\mathrm{X}$ - "Do you bath/play games when you visit the water body in your community"?

$Y$ - "Have you been passing blood in your urine recently"?

Z - "Do you feel pains when urinating"?

Table 5. Comparison of intensity of infection and responses to questionnaire

\begin{tabular}{|c|c|c|c|c|c|c|c|c|}
\hline \multirow[t]{2}{*}{ Intensity, eggs $/ 10 \mathrm{ml}$ of urine } & \multicolumn{2}{|c|}{$W$, no. (\%); $N=417$} & \multicolumn{2}{|c|}{$X, n o .(\%) ; N=417$} & \multicolumn{2}{|c|}{$Y$, no. (\%); $N=417$} & \multicolumn{2}{|c|}{$Z$, no. (\%); $N=417$} \\
\hline & Yes & No & Yes & No & Yes & No & Yes & No \\
\hline$<50$ & $47(11.3)$ & $22(5.2)$ & $19(4.6)$ & $50(12.0)$ & $40(9.6)$ & $29(7.0)$ & $35(8.4)$ & $34(8.2)$ \\
\hline$\geq 50$ & $13(3.1)$ & $5(1.2)$ & $3(0.7)$ & $15(4.3)$ & $9(2.2)$ & $9(2.2)$ & $8(1.9)$ & $10(2.4)$ \\
\hline No ova seen & 174 (41.7) & $15637.4)$ & $102(24.5)$ & 228 (53.4) & 131 (31.4) & 199 (47.7) & $122(29.3)$ & 208 (49.9) \\
\hline
\end{tabular}

WXYZ represents selected questions analyzed for this manuscript.

Table 6. Sensitivity and specificity estimations for selected questions

\begin{tabular}{ccccc}
\hline Question & Sensitivity, \% & Specificity, \% & $P P V, \%$ & $N P V, \%$ \\
\hline Y & 56.3 & 60.3 & 27.2 & 84.0 \\
Z & 49.4 & 63.0 & 26.1 & 82.5 \\
\hline
\end{tabular}

PPV, Positive Predictive Value; NPV, Negative Predictive Value.

2. X - "Do you bath/play games when you visit the water body in your community"?

With regards to water contact, which describes Prevalence of Exposure, 29.7\% (124/417) responded "yes" to bathing and playing games in a water body in their communities. Out of this number, $17.7 \%(22 / 124)$ had S. haematobium parasite ova in their urine. Out of the total number of participants, 293 responded that they do not bath or play games when they visit the water body in their various communities. Out of this number $22.2 \%$ (65/293) had parasite ova in their urine (Table 4).

3. Y - "Have you been passing blood in your urine recently"?

With regards to the above question, 43.2\% (180/417) responded "yes" to passing blood in their urine in recent times (Table 4). Out of this number, $27.2 \%(49 / 180)$ had parasite ova in their urine. In this category, 237 responded "no" to passing blood in their urine, out of which $16.0 \%(38 / 237)$ were positive for parasite ova ( $\mathrm{POR}=0.7566, \mathrm{Cl}: 0.43-0.80$ ). There was a significant difference between female $(16.1 \%, 67 / 417)$ and male $(27.1 \%$, $113 / 417)$ responses to self reported haematuria $\left(\chi^{2}=4.170\right.$, $\mathrm{p}<0.001)$.

Of the 180 participants who had been passing blood in their urine, $58.9 \%(106 / 180)$ through further responses to other questions (data not shown), visited the hospital/health centre for treatment. In addition $10.0 \%(18 / 180)$ reported using herbal medicine, which they couldn't mention, $11.7 \%$ (21/180) used medicine bought from the drug store, $11.7 \%(21 / 180)$ did nothing about it, while $7.8 \%(14 / 180)$ did not respond to the question.

\section{Z - "Do you feel pains when urinating"?}

Concerning painful urination, $39.6 \%(165 / 417)$ responded in the affirmative. The remaining 252 participants responded not feeling any pains while urinating (Table 4). Out of this number, $17.5 \%(44 / 252)$ had parasite ova in their urine.

When participants were asked what they did about the pain experienced while urinating, $62.4 \%$ (103/165) visited the hospital / health centre, $9.7 \%(16 / 165)$ used herbal medicine, which they couldn't name, $10.9 \%(18 / 165)$ used medicine bought from the drug store, $12.1 \%(20 / 165)$ did not do anything about it, while $4.8 \%$ (8/165) didn't respond to the question (data not shown).

Intensity categorization and results from individuals with 'no ova seen' in their urine was cross tabulated with the outcomes of the selected questions (Table 5). In questions ' $Y$ ' and ' $\mathrm{Z}$ ', although $31.4 \%$ (131/417) and 29.3\% (122/417) confirmed urinating blood and painful urination respectively, there was no parasite ova seen in their urine. Of the total, $7.0 \%(29 / 417)$ and 2.2\% (9/417) responded not seeing blood in their urine but had low and high intensity respectively (Question $\mathrm{Y}$ ). With regards to painful urination (Question Z), 8.2\% (34/417) and 2.4\% (10/417) answered 'no' to painful urination but had low and high intensity infection respectively.

\section{Sensitivity and specificity of questions ' $Y$ ' and ' $Z$ '}

Microscopy results were compared with answers from questions ' $Y$ ' and ' $Z$ ' to estimate the appropriateness of questionnaire use in determining prevalence (Table 6). Questions ' $Y$ ' and ' $Z$ ' respectively identified correctly, $56.3 \%$ and $49.4 \%$ of individuals positive by microscopy. With regards to specificity, ' $Y$ ' and ' $Z$ ' respectively wrongly identified $39.7 \%$ and $37.0 \%$ as being 
infected with S. haematobium. There is only $27.2 \%$ and $26.1 \%$ chance for ' $Y$ ' and ' $Z$ ' respectively to correctly identify that anyone who responds 'yes' by questionnaire has ova in his or her urine (PPV). Also, there is $84.0 \%$ and $82.5 \%$ chance for questions ' $Y$ ' and ' $\mathrm{Z}$ ' respectively, that anyone who responds ' $\mathrm{no}$ ' to urinating blood has no ova in urine (NPV).

\section{Discussion}

Prevalence and intensity estimates from microscopy are extrapolated to establish infection trends in entire communities, and to influence nationwide control strategies. However, limited resources and technical challenges faced have led to the use of urine chemistry reagent strips and questionnaire for establishing preliminary data for mass drug administration.

A prevalence of $20.9 \%$ was established from microscopy in this study, with no significant difference among males and females $\left(\chi^{2}=2.582, p=0.108\right)$. This overall prevalence is categorized as 'moderate' by the World Health Organization (WHO) [4], which recommends that both enrolled and non-enrolled children in the study area are treated once every two years. In Ghana, treatment regimens have been based on foreign sponsorships and are therefore not consistent over the years. A significant proportion $\left(\chi^{2}=22.442, p<0.001\right)$ of prevalence $(14.0 \%)$ was however found among the 10-15 years age group. There is an existing trend of high infectivity among this age group in Ghana and other Sub Saharan African countries due to their exploratory nature, especially when they have the freedom to play around in water bodies located in rural communities.

Prevalence due to macrohaematuria alone (3.6\%) and the use of urine chemistry reagent strips gave varying treatment recommendations compared to that of microscopy. Their sensitivities were also low but with appreciable specificities. Leucocyturia singled out to have the highest sensitivity but with zero specificity, since all the participants were positive with varying categorization (+ and ++ ). This study shows that the use of leucocyturia alone is grossly inappropriate as a morbidity indicator for urinary schistosomiasis. A consolidated average of $11.7 \%$ of participants who had ova in their urine, had haematuria, leucocyturia or proteinuria. Significantly, of ova positive individuals, $86.2 \%$ had no macrohaematuria, $33.3 \%$ had no proteinuria and $48.3 \%$ had no microhaematuria. Clearly most infected participants had no morbidity signs, making it obvious that decision making should not be based entirely on presumptive diagnosis.

Earlier studies elsewhere have suggested that the combination of proteinuria and haematuria $[14,15]$ or a combination of proteinuria, haematuria and leucocyturia readings [16] may increase the sensitivity and specificity of the detection of S. haematobium infection. Results from this study are suggestive that these morbidity indicators and their combinations are not sufficient for screening purposes that are meant for decision making towards treatment.

Gross haematuria in school-age children is a widely recognized clinical manifestation of $S$. haematobium infection. In this current study, visual inspection of the urine specimens identified only $2.9 \%$ of the infected children. No studies of entire communities in endemic areas in Ghana are available to indicate the proportion of cases of haematuria, which may be due to other causes. Only $8.0 \%$ had macrohaematuria with heavy intensity, while $5.7 \%$, also with macrohaematuria were lightly infected. The low frequency of gross haematuria from infected children indicates that other causes may be relatively infrequent. This current study is contrary to the established effectiveness of the use of morbidity indicators for control programmes to reduce morbidity due to $S$. haematobium infection.

A total of 25 infected individuals did not have any form of haematuria and proteinuria. Out of these, one pupil had heavy intensity ( 80 eggs $/ 10 \mathrm{ml}$ of urine) while the remaining 24 were of low intensity infection (1-44 eggs/10ml of urine). Leucocyturia was common among all pupils infected. In low resourced countries, including Ghana, low intensity leucocyturia is considered normal. However, medical laboratory reports that indicates about 20-30 pus cells (leucocytes) is interpreted as urinary tract infection, where in the absence of microbiological culturing facilities, are treated with broad spectrum antibiotics.

Existing information in endemic areas around the Volta Lake in Ghana showsup to $30.0 \%$ of $S$. haematobium infections with 2 eggs $/ 5 \mathrm{ml}$ of urine going undetected by examination of a single urine specimen, using the filtration method [17]. This current study doubled the urine volume to $10 \mathrm{ml}$ of urine and was able to detect as low as $1 \mathrm{egg} / 10 \mathrm{ml}$ of urine in $25.3 \%$ of infected participants. Future studies could increase volumes of urine provided by study participants to observe the outcomes.

From the four main questions considered for analysis, $42.2 \%$ knew that passing blood in urine is a disease. The remaining didn't know of the major characteristic symptom of $S$. haematobium infection, although they were living in an endemic area. This is quite significant because $14.9 \%$ (36/241) of this number remaining were positive by microscopy and were passing bloody urine or had previously passed bloody urine. Extensive school and community health education must be intensified to increase knowledge and health seeking behavior among schools and the general populace in endemic rural communities.

Prevalence based on exposure was estimated to be $29.7 \%$, where $17.7 \%$ were positive by microscopy. Among the 293 who disclaimed any water contact, $22.2 \%$ had ova seen in their urine. This is evident from the biases that have been described from various questionnaire studies elsewhere. Improved ways of questioning and education must be employed to draw relevant answers from participants in future primary school studies, without influencing their choice.

Of the total number of participants, $43.2 \%$ responded seeing blood in their urine. This is categorized as high prevalence according to the WHO Technical Report Series [4]. Out of this number, $27.2 \%$ were also ova positive. Both percentages obtained are greater than the overall microscopy prevalence of $20.9 \%$. Over-reliance on prevalence by questionnaire could lead to overapplication of control strategies in peri-urban areas, although in our view it could be beneficial for rural communities in low resourced countries where control strategies are mostly sponsored. It is worth noting that out of 237 pupils who had answered 'no' to passing blood in their urine recently, $16.8 \%$ were ova positive. Of the 108 who responded 'yes' to passing bloody urine, 58.9\% (106/180) had sought medical treatment from a hospital/health centre. The remaining $41.1 \%$ reported diverse treatment options, with some doing virtually nothing about the situation. We recommend studies involving adults, where questions relating to their past, recent and future local treatment options in their communities will be documented. Pharmacokinetics of local herbal remedies to urinary 
schistosomiasis should be investigated for refinement and subsequent trials.

Findings from studies among Kenyan school children had revealed that prevalence of self-reported haematuria was lower among females than among males of all ages [11]. This is in consonance with this study which also found a significant difference between female (67/417) and male (113/417) responses to self reported haematuria $\left(\chi^{2}=4.170, p<0.001\right)$. Females at their pubertal ages may probably consider haematuria as normal, as they are educated locally through pubertal initiation rituals, compared to males who will find it very strange and probably dreaded, making males seek medical attention.

With regards to painful urination, $39.6 \%$ responded in the affirmative with $43 / 165$ being positive by microscopy. Remarkably, of the 252 who responded 'no' to painful urination, $17.5 \%$ had parasite ova in their urine. Possibly these participants were not experiencing the symptom, or did not provide the right answer. This is so, although questions were explained in their local language by teachers of the participating schools and community field workers. With treatment seeking behavior, $62.4 \%$ who had painful urination visited the hospital/health centre. Others, 9.5\%, who used herbal medicine could not name the type of herbal preparations and quantities prescribed. Further studies should ask adults of the type of local preparations they use for the treatment of painful urination.

The sensitivity and specificity of questionnaire was estimated alongside the gold standard to compare its effectiveness in determining the WHO criteria for treatment in rural and periurban areas. This was done using questions on haematuria $(\mathrm{Y})$ and painful urination (Z). Sensitivity and specificity estimates for both were low and cannot be relied on to determine the WHO criteria for treatment. The study however estimated an NPV of $84.0 \%$ and $82.5 \%$ for $Y$ and $Z$ respectively. Therefore, every pupil who responded 'no' to passing blood in urine has $84.0 \%$ chance of not passing ova in urine. Likewise, there was $82.5 \%$ chance of not passing out ova if the response to painful urination was 'no'. It was estimated that the probability of being ova positive by microscopy, following a 'yes' response to haematuria or painful urination is $<30.0 \%$ (PPV). There is a direct association between the PPV obtained and the percentage of participants who were ova positive, although it is not directly dependent. Future studies may have to modify how questions are explained to study participants and consider retraining teachers and community volunteers who greatly support such studies.

\section{Conclusion}

Screening procedures focused on morbidity indicators alone will not be suitable to recognize a large fraction of persons infected by S. haematobium in defined populations. In addition, questionnaire though useful, might not be appropriate in establishing existing control strategies and treatment guidelines from the WHO. It could however be useful for surveillance purposes.

\section{Conflict of interest}

The authors declare that there is no conflict of interest.

\section{Acknowledgements}

We express our sincere thanks to our collaborators, mainly, the Director and officers of Ghana Education Service, Dangme East District, the disease control officer of the Kasseh Health Centre and Mrs. Comfort Ametame.

\section{References}

1. Bosompem KM, Asigbee JRK, Otchere J, Haruna A, Kpo HK, Kojima S. Accuracy of diagnosis of urinary schistosomiasis: comparison of parasitological and a monoclonal antibody-based dipstick. Parasitology International 1998; 47: 211-217. (doi:10.1016/S1383-5769(98)00021$\mathrm{X})$

2. Aryeetey ME, Wagatsuma Y, Yeboah GK, Asante M, Mensah G, Nkrumah FK, Kojima S. Urinary schistosomiasis in southern Ghana: Prevalence and morbidity assessment in three (defined) rural areas drained by the Densu River. Parasitology International 2000; 49: 155 163. (doi:10.1016/S1383-5769(00)00044-1) (PMID: 10882905)

3. Vector control. Methods for use by individuals and communities. Chapter 8. WHO (World Health Organization), 1997.

4. Prevention and control of schistosomiasis and soil-transmitted helminthiasis. Geneva: WHO Technical Series, Report 912. WHO (World Health Organization), 2002.

5. Ansell J, Guyatt HL, Hall A, Kihamia C, Kivogo J, Ntimbwa P, Bundy D. The reliability of self reported blood in urine and schistosomiasis as indicators of Schistosoma haematobium in school children: a study in Muheza district, Tanzania. Trop Med Int Health 1997; 2: 1180-1189. (doi: 10.1046/j.1365-3156.1997.d01-209.x) (PMID: 9438475)

6. Mafe MA. The diagnostic potential of three indirect tests for urinary schistosomiasis in Nigeria. Acta Tropica 1997; 68: 277-284. (doi: 10.1016/S0001-706X(97)00102-2)

7. Anosike JC, Nwoke BEB, Njoku AJ. The validity of haematuria in the community diagnosis of urinary schistosomiasis infection. Journal of Helminthology 2001; 75: 223-225. (doi: 10.1079/JOH200048)

8. Bosompem KM, Owusu O, Okanla EO, Kojima S. Applicability of a monoclonal antibody-based dipstick in diagnosis of urinary schistosomiasis in the Central Region of Ghana. Trop Med Int Health 2004; 9: 991-996. (doi: 10.1111/j.1365-3156.2004.01289.x) (PMID: 15361112)

9. Lengeler C, de Savigny D, Mshinda H, Mayombana C, Tayari S, HatzDegremont CA, Tanner M. Community-based questionnaires and health statistics as tools for the cost-efficient identification of communities at risk of urinary schistosomiasis. Int J Epidemiol 1991; 20: 796-807. (doi: 10.1093/ije/20.3.796) (PMID: 1955266)

10. Lengeler C, Makwala J, Ngimbi D, Utzinger J. Simple school questionnaires can map both Schistosoma mansoni and Schistosoma haematobium in the Democratic Republic of Congo. Acta Tropica 2000; 74: 77-87. (doi: 10.1016/S0001-706X(99)00046-7) (PMID: 10643911)

11. Kihara J, Mwandawiro C, Waweru B, Gitonga CW, Brooker S. Preparing for national school-based deworming in Kenya: the validation and large-scale distribution of school questionnaires with urinary schistosomiasis. Trop Med Int Health 2011; 16: 1365-3156. (doi: 10.1111/j.1365-3156.2011.02829.X)

12. Peters PA. Rapid accurate quantification of schistosome eggs via nuclepore filters. Journal of Parasitology 1976; 62: 154-155. (PMID: 1255368)

13. Cheesbrough M. District laboratory practice in tropical countries. Part 1. Cambridge: Cambridge University Press, 2005; 454 p.

14. Wilkins HAP, Goll C, Marshal De, Moore P. The significance of proteinuria and haematuria in Schistosoma haematobium infection. Trans R Soc Trop Med Hyg 1979; 73: 74-80. (PMID: 442186)

15. Pugh RNH, Bell DR, Gilles HM. Malumfashi Endemic Diseases Research Project, XV. The potential medical importance of bilharzia in northern Nigeria: a suggested rapid, cheap and effective solution for control of 
Schistosoma haematobium infection. Ann Trop Med Parasitol 1980; 74: 597-613. (PMID: 7458464)

16. Feldmeier H, Doehring E, Daffalla AA. Simultaneous use of a sensitive filtration technique and reagent strips in urinary schistosomiasis. Trans $R$ Soc Trop Med Hyg 1982; 76: 416-421. (doi: 10.1016/00359203(82)90204-8)

17. Scott D, Senker K, England EC. Epidemiology of human Schistosoma haematobium infection around the Volta Lake, Ghana, 1973-1975. Bull World Health Organ 1982; 60: 89-100. (PMID: 6979420)

Authors:

Ato Kwamena Tetteh - MPHIL, Lecturer, Department of Community Medicine and Health, Anglican University College of Technology, Nkoranza, Brong Ahafo Region, Ghana.

Joseph Otchere - MPHIL, Chief Research Assistant, Department of Parasitology, Noguchi Memorial Institute for Medical Research University of Ghana, Legon, Accra, Ghana.

Langbong Bimi - PhD, Associate Professor, Department of Animal Biology and Conservation Sciences, University of Ghana, Legon, Accra, Ghana.

Irene Ayi - PhD, Snr. Research Fellow, Department of Parasitology, Noguchi Memorial Institute for Medical Research University of Ghana, Legon, Accra, Ghana. 
Date.

ID No.

\section{QUESTIONNAIRE ON SCHISTOSOMIASIS}

Name of pupil (Optional)

Age

Sex M [ ] F [ ]

School.

Class

Community

House Number

[Tick $[\sqrt{ }]$ where appropriate]

\section{SCHISTOSOMIASIS (BILHARZIA)}

1. Do you stay outside this community sometimes? Yes [ ] No [ ]

2. If 'Yes' to question 1 above, where do you go?

3. Do you come into contact with any water body (river, pond, lake, etc.) when you travel?

Yes [ ] No [ ]

4. If 'Yes' to question 3 above, what is the name of the water body?

Don't know [ ]

5. Do you come into contact with any water body (river, pond, lake, etc.) in your community

Yes [ ] No [ ]

6. If 'Yes' to question 5 above, what is the name of this water body?

Don't know [ ]

7. What do you do when you visit this water body? (Tick the most appropriate; multiple choices allowed)

a. bath, play games etc [ ]

b. fetch water ( for drinking, washing, farming etc) [ ]

c. catch fish from it [ ]

d. other (specify)

8. How often do you come into contact with this water body?

a. daily [ ]

b. one to three times per week [ ]

c. once a month [ ]

d. Twice a year [ ]

e. never [ ]

9. Do you accompany any of your parents to fish in a river, pond etc.? Yes [ ] No [ ]

10. Do you accompany any of your parents to the farm/the garden? Yes [ ] No [ ] 
If yes to question 10 above, answer question 11

11. Do you cross a pond, river etc. when going to the farm/garden? Yes [ ] No [ ]

12. Do you know Bilharzia? Yes [ ] No [ ]

If yes to question 12 above, answer question 13

13. What do you think it is?

a. sign of reproductive maturity [ ]

b. punishment from gods/ancestors [ ]

c. a disease [ ]

d. don't know [ ]

e. other (please specify)

14. There are two forms of Bilharzia. True [ ] False [ ] Don’t know [ ]

15. Which of the following is true about Bilharzia?

a. chills/shivering [ ]

b. swollen stomach [ ]

c. passing blood in urine [ ]

d. feeling pains when urinating [ ]

e. passing blood in stool [ ]

16. Have you been passing blood in your urine recently? Yes [ ] No [ ] don't know [ ]

If yes to question 16 above, answer question 17

17. Do you feel pains when urinating? Yes [ ] No [ ]

18. Have you ever passed blood in your stool? Yes [ ] No [ ] Don't know [ ]

19. If yes to any of questions 16,17 or 18 what was done about it?

a. visited a hospital/health centre for treatment [ ]

b. used herbal medicine [ ]

c. used medicine bought from a drug store [ ]

d. did not do anything about it [ ]

e. Other (specify)

If (a) in question 19 is ticked answer question 20

20. When was the last time you received the treatment?

Up to 6 months ago [ ]

Up to one year ago [ ]

Don't remember [ ] 